\title{
Vitamin D Pathway Genes, Diet, and Risk of Renal Cell Carcinoma
}

\author{
S. Karami, ${ }^{1}$ P. Brennan, ${ }^{2}$ M. Navratilova, ${ }^{3}$ D. Mates, ${ }^{4}$ D. Zaridze, ${ }^{5}$ V. Janout, ${ }^{6}$ \\ H. Kollarova, ${ }^{6}$ V. Bencko, ${ }^{7}$ V. Matveev, ${ }^{5}$ N. Szesznia-Dabrowska, ${ }^{8}$ I. Holcatova, ${ }^{7}$ M. Yeager, ${ }^{9}$ \\ S. Chanock, ${ }^{9}$ N. Rothman, ${ }^{1}$ P. Boffetta, ${ }^{2}$ W-H. Chow, ${ }^{1}$ and L. E. Moore ${ }^{1}$ \\ ${ }^{1}$ Division of Cancer Epidemiology and Genetics, National Cancer Institute, NIH, DHHS, Bethesda, MD, USA \\ ${ }^{2}$ International Agency for Research on Cancer, Lyon, France \\ ${ }^{3}$ Department of Cancer Epidemiology and Genetics, Masaryk Memorial Cancer Institute, Brno, Czech Republic \\ ${ }^{4}$ Institue of Public Health, Bucharest, Romania \\ ${ }^{5}$ Institute of Carcinogenesis, Cancer Research Centre, Moscow, Russia \\ ${ }^{6}$ Department of Preventive Medicine, Faculty of Medicine, Palacky University, Olomouc, Czech Republic \\ ${ }^{7}$ Institute of Hygiene and Epidemiology, First Faculty of Medicine, Charles University in Prague, Czech Republic \\ ${ }^{8}$ Department of Epidemiology, Institute of Occupational Medicine, Lodz, Poland \\ ${ }^{9}$ Core Genotyping Facility, Advanced Technology Center, Bethseda National Cancer Institute, NIH, \\ Department of Health and Human Services, MD, USA
}

Correspondence should be addressed to S. Karami, karamis@mail.nih.gov

Received 31 March 2009; Accepted 6 August 2009

Academic Editor: Diane Kamen

Copyright (๑) 2010 S. Karami et al. This is an open access article distributed under the Creative Commons Attribution License, which permits unrestricted use, distribution, and reproduction in any medium, provided the original work is properly cited.

\begin{abstract}
Mediated by binding to the high-affinity vitamin D receptor (VDR), vitamin D forms a heterodimer complex with the retinoid$\mathrm{X}$-receptor (RXR). Variation in both genes has been shown to modify renal cell carcinoma (RCC) risk. Therefore, we investigated whether VDR and RXRA polymorphisms modify associations between RCC risk and frequency of dietary intake of vitamin D and calcium rich foods, and occupational ultraviolet exposure among 777 RCC case and 1035 controls from Central and Eastern Europe. A positive association was observed in this population between increasing dietary intake frequency of yogurt, while an inverse association was observed with egg intake frequency. RXRA polymorphisms, located $3^{\prime}$ of the coding sequence, modified associations between specific vitamin D rich foods and RCC risk, while RXRA polymorphisms, located in introns 1 and 4, modified associations with specific calcium rich foods. Results suggest that variants in the RXRA gene modified the associations observed between RCC risk and calcium and vitamin D intake.
\end{abstract}

\section{Introduction}

Smoking, obesity, and hypertension are well-established risk factors of renal cell carcinoma (RCC) $[1,2]$. Other risk factors such as dietary practices have been implicated, though inconsistent results have usually been reported for specific dietary components $[1,3]$. Diets rich in fruits and vegetables however have typically been shown to decrease RCC risk possibly through antioxidant effects, while consumption of fried foods has commonly been shown to increase risk possibly due to the potential carcinogenic effects of acrylamides [1-3]. Recently, epidemiological studies suggest that vitamin $\mathrm{D}$, which is found in food (vitamin $\mathrm{D}_{2}$ and $\mathrm{D}_{3}$ ) and produced in the body after exposure to ultraviolet (UV) rays from the sun (vitamin $\mathrm{D}_{3}$ ), may be inversely associated with RCC risk [4-7].

Both vitamin $\mathrm{D}_{2}$ and $\mathrm{D}_{3}$ are hydroxylated in the liver and subsequently in the kidney to form active vitamin $\mathrm{D}$ $\left(1,25(\mathrm{OH})_{2} \mathrm{D}_{3}\right)[8]$. Dietary intake of vitamin $\mathrm{D}$ accounts for a small (approximately 10\%) proportion of vitamin D levels [8-10]. However, dietary intake of vitamin D and vitamin D rich foods may play a role in determining RCC risk since vitamin $\mathrm{D}$ has been associated with anticarcinogenic properties and is primarily metabolized within the kidneys $[8,11]$.

Although the exact anticarcinogenic mechanism of vitamin D is not fully understood, vitamin D and its metabolites 
are thought to impede carcinogenesis by stimulating cell differentiation, inhibiting cell proliferation, inducing apoptosis, and suppressing invasiveness, angiogenesis, and metastasis [11-14]. Vitamin D is mediated by binding to vitamin D receptors (VDRs), transcription factors that are part of the nuclear hormone receptor family. Forming a heterodimer complex with the retinoid-X-receptor $(R X R)$ gene, VDR can regulate the transcription of other genes involved in cell regulation, growth, and immunity $[13,15,16]$. Most epidemiological studies have generally focused on the VDR gene, however recently, we evaluated 139 single nucleotide polymorphisms (SNPs) across eight genes of the vitamin D pathway and found a significant association between RCC risk and across certain $V D R$ and $R X R A$ genetic variants [17].

Given that the kidney is the most important organ for vitamin D metabolism and activity and calcium homeostasis and that within the kidneys, calcium has been shown to influence active vitamin D levels $[8,17]$, investigation of dietary vitamin $\mathrm{D}$ and calcium in RCC etiology are highly relevant. We therefore investigated whether common genetic variation in $V D R$ and $R X R A$, the two genes we observed to be significantly associated with RCC risk, modified associations between renal cancer risk and the frequency of dietary intake of vitamin $\mathrm{D}$ and calcium rich foods. Additionally, we also explored whether these genes modified occupational UV exposure and RCC risk, since recently we reported a significant inverse association between occupational UV exposure and RCC risk among male participants in this population [18] and because the majority of people worldwide obtain most of their vitamin D levels from the sun [8-10]. Analyses were conducted among cases and controls from Central and Eastern Europe, an area with one of the highest rates of RCC worldwide and an area where foods are not regularly fortified with either vitamin D or calcium [19].

\section{Materials and Methods}

2.1. Study Population. Details regarding this study population were previously described [20]. Briefly, from 1999 through 2003, a hospital-based case-control study of RCC was conducted in seven centers in four countries of Central and Eastern Europe (Moscow, Russia; Bucharest, Romania; Lodz, Poland; Prague, Olomouc, Ceske-Budejovice, and Brno, Czech Republic). Cases, 20 to 88 years of age, included newly diagnosed patients (within 3 months) with histologically confirmed RCC (IDC-O-2 codes C64) who resided in the study areas for at least one year. Controls in all centers were frequency matched to cases on age $( \pm 3$ years), sex, and place of residence. Controls were chosen among subjects admitted as inpatients or out patients in the same hospital as the cases, with non-tobacco-related conditions, including infections (1.1\%), hematologic (3.2\%), endocrine $(2.0 \%)$, psychiatric $(1.4 \%)$, neurologic $(11.2 \%)$, ophthalmologic or otologic (14.5\%), cardiovascular (9.6\%), pulmonary $(3.9 \%)$, gastrointestinal $(18.7 \%)$, dermatologic $(2.8 \%)$, orthopedic or rheumatologic $(8.9 \%)$, genitourinary (benign prostatic hyperplasia) (3.8\%), obstetric or perinatal $(0.1 \%)$, injury or poisoning $(3.0 \%)$, and other $(15.9 \%)$. No single disease made up more than $20 \%$ of the control group. Furthermore, a number of controls were also recruited in parallel for studies of lung and head and neck cancers $[21,22]$. A total of 1097 incident, histologically confirmed RCC cases and 1476 controls were included in this study. Suitable quantity and quality of genomic DNA was obtained from a subset of $777(70.8 \%)$ eligible RCC cases and 1035 $(70.1 \%)$ controls enrolled in our study. The response rates across study centers for study participation ranged from $90.0 \%$ to $98.6 \%$ for cases and from $90.3 \%$ to $96.1 \%$ for controls. All subjects provided written informed consent. This study was approved by the institutional review boards of all participating centers.

2.2. Dietary Intake. Details of dietary assessment have been previously described [23]. Briefly, interviewers were trained in each center to perform face-to-face interviews with cases and controls during hospitalization using standard questionnaires. The questionnaire covered demographic characteristics, family history of cancer, history of tobacco consumption, and dietary habits. A food frequency questionnaire was comprised of 23 food items, which the study investigators selected by consensus during the planning stage of the study and further validated during the pilot stage by asking participants to name common food items not already specified [23]. Frequency of dietary intake for each food group was assessed for each item as never, less than once per month, less than once per week, one to two times per week, three to five times per week, and daily. A standardized questionnaire was used in each of the study centers that was translated from a common English version and then backtranslated into English to ensure the validity of the initial translation. The questionnaire was repeated for two different time periods: (1) the year prior to interview, and (2) the year prior to political and market changes in 1989 (1991 in Russia). Data for the year prior to interview and the year prior to political change were then extrapolated to represent lifetime average dietary intake by multiplying the score for each time period by the number of years the participant was alive during the time period, then summing the time period scores and dividing by the total age of the individual as previously described.

In this study, intake frequencies of vitamin $\mathrm{D}$ rich foods which include liver, eggs, and fish were combined for each participant to create a new dietary exposure variable for total vitamin D intake frequency. Since calcium has been reported to influence vitamin $\mathrm{D}$ levels and risk of certain cancers [8], intake frequencies of cheese, milk, and yogurt were also combined for each participant to create a new total calcium intake frequency exposure variable. In this study, specific details regarding the type of fish, cheese, milk, and so forth consumed was not available; therefore, each food category was assumed to have equal weights when combined to estimate total vitamin D or total calcium lifetime average dietary intake frequency. Very few foods naturally contain vitamin D and in the United States (US) and Canada, fortified foods are common sources of vitamin $\mathrm{D}[8,9]$. However, in most countries, such as those in Central and 
TABLE 1: General characteristics of genotyped participants.

\begin{tabular}{|c|c|c|c|c|c|}
\hline \multirow{3}{*}{ Variables } & \multicolumn{4}{|c|}{ Genotyped participants } & \multirow[b]{3}{*}{${ }^{\dagger} P$-value } \\
\hline & \multicolumn{2}{|c|}{ Cases } & \multicolumn{2}{|c|}{ Controls } & \\
\hline & $\mathrm{N}$ & $\%$ & $\mathrm{~N}$ & $\%$ & \\
\hline Participants & 777 & 42.9 & 1,035 & 57.1 & \\
\hline \multicolumn{6}{|l|}{ Sex } \\
\hline Males & 472 & 60.7 & 648 & 62.6 & \\
\hline Females & 305 & 39.3 & 387 & 37.4 & .42 \\
\hline \multicolumn{6}{|l|}{ Age at Interview } \\
\hline$<45$ & 60 & 7.7 & 83 & 8.0 & \\
\hline $45-54$ & 197 & 25.4 & 287 & 27.7 & \\
\hline $55-64$ & 243 & 31.3 & 309 & 29.9 & \\
\hline $65-74$ & 242 & 31.1 & 318 & 30.7 & \\
\hline $75+$ & 35 & 4.5 & 38 & 3.7 & .30 \\
\hline Mean age (std) & \multicolumn{2}{|c|}{59.5 years $(10.4)$} & \multicolumn{2}{|c|}{59.0 years $(10.2)$} & \\
\hline \multicolumn{6}{|l|}{ Center } \\
\hline Romania-Bucharest & 68 & 8.8 & 94 & 9.1 & \\
\hline Poland-Lodz & 80 & 10.3 & 189 & 18.3 & \\
\hline Russia-Moscow & 242 & 31.1 & 313 & 30.2 & \\
\hline${ }^{*}$ Czech Republic & 387 & 49.8 & 439 & 42.4 & $<.001$ \\
\hline \multicolumn{6}{|l|}{ BMI at Interview } \\
\hline$<25$ & 222 & 28.6 & 375 & 36.3 & \\
\hline $25-29.9$ & 330 & 42.5 & 432 & 41.9 & \\
\hline $30+$ & 225 & 29.0 & 225 & 21.8 & $<.001$ \\
\hline \multicolumn{6}{|l|}{ Tobacco status } \\
\hline Never & 359 & 46.4 & 420 & 40.7 & \\
\hline Ever & 415 & 53.6 & 613 & 59.3 & .02 \\
\hline \multicolumn{6}{|l|}{ Hypertension } \\
\hline No & 434 & 55.9 & 638 & 61.7 & \\
\hline Yes & 342 & 44.1 & 396 & 38.3 & .01 \\
\hline \multicolumn{6}{|c|}{$\begin{array}{l}\text { Familial history of cancer } \\
\text { Among 1st degree relatives }\end{array}$} \\
\hline No & 512 & 65.9 & 745 & 72.0 & \\
\hline Yes & 265 & 34.1 & 290 & 28.0 & .01 \\
\hline
\end{tabular}

* Brno, Olomouc, Prague, Ceske-Budejovice; ${ }^{\dagger}$ Crude $P$-values.

Eastern Europe, foods are not fortified with vitamin D or calcium. Therefore the major sources of vitamin D intake are fatty fish, such as mackerel and salmon, while the main source of calcium intake is primarily dairy products [7-9]. All dietary intake frequencies in this study were assessed in tertiles based on the frequency of dietary intake among controls.

2.3. Occupational UV Exposure. Lifetime occupational information for jobs held for at least 12 months duration was also ascertained during interviews through the use of a general occupational questionnaire. Data collected for each job included title, detailed tasks, and type of employer as well as the year of beginning and ending employment. These data were used to create a job-exposure matrix (JEM) which classified each study subjects' estimated occupational exposure to sunlight; the JEM is described in more detail elsewhere [18]. To assess the relationship between estimated occupational sunlight exposure, RCC risk and variation in vitamin $\mathrm{D}$ pathway genes, cumulative exposure across all jobs (calculated as duration (years) $\mathbf{x}$ frequency midpoint $\mathbf{x}$ intensity of exposure for each job and summed over all jobs held) was assessed. Since the association between occupational UV exposure and RCC risk in this population was previously observed only among males [18], evaluation of genetic variants, occupational UV exposure, and RCC risk are only presented for male participants. The relationship between these exposures and RCC risk was also explored among female participants; however no association was observed (data not shown). 
TABLE 2: RCC risk by dietary intake frequency of vitamin D and calcium rich foods among genotyped participants.

\begin{tabular}{|c|c|c|c|c|c|c|c|c|c|}
\hline \multirow[b]{2}{*}{ Intake Frequency } & \multicolumn{2}{|c|}{ Cases } & \multicolumn{2}{|c|}{ Controls } & \multicolumn{5}{|c|}{ Adjusted } \\
\hline & $\mathrm{N}$ & $\%$ & $\mathrm{~N}$ & $\%$ & OR & UCI & - & LCI & $P$-trend \\
\hline \multicolumn{10}{|l|}{ Liver } \\
\hline Low $(<33 \%)$ & 318 & 41.0 & 411 & 39.7 & 1.00 & & & & \\
\hline Medium (33-66\%) & 315 & 40.6 & 380 & 36.7 & 1.11 & 0.89 & - & 1.38 & \\
\hline High $(>66 \%)$ & 143 & 18.4 & 244 & 23.6 & 0.82 & 0.63 & - & 1.07 & .28 \\
\hline \multicolumn{10}{|l|}{ Egg } \\
\hline Low $(<33 \%)$ & 274 & 35.3 & 345 & 33.3 & 1.00 & & & & \\
\hline Medium (33-66\%) & 392 & 50.5 & 454 & 43.9 & 1.11 & 0.90 & - & 1.38 & \\
\hline High $(>66 \%)$ & 110 & 14.2 & 236 & 22.8 & 0.64 & 0.48 & - & 0.86 & .02 \\
\hline \multicolumn{10}{|l|}{ Fresh Water Fish } \\
\hline Low $(<33 \%)$ & 260 & 33.5 & 345 & 33.3 & 1.00 & & & & \\
\hline Medium (33-66\%) & 274 & 35.3 & 344 & 33.2 & 1.06 & 0.84 & - & 1.34 & \\
\hline High $(>66 \%)$ & 242 & 31.2 & 346 & 33.4 & 0.94 & 0.74 & - & 1.19 & .60 \\
\hline \multicolumn{10}{|l|}{ Salt Water Fish } \\
\hline Low $(<33 \%)$ & 259 & 33.4 & 346 & 33.4 & 1.00 & & & & \\
\hline Medium (33-66\%) & 334 & 43.0 & 419 & 40.5 & 1.02 & 0.82 & - & 1.27 & \\
\hline High $(>66 \%)$ & 183 & 23.6 & 270 & 26.1 & 0.91 & 0.71 & - & 1.18 & .53 \\
\hline \multicolumn{10}{|l|}{ Total Fish } \\
\hline Low $(<33 \%)$ & 290 & 37.4 & 380 & 36.7 & 1.00 & & & & \\
\hline Medium (33-66\%) & 236 & 30.4 & 309 & 29.9 & 0.98 & 0.77 & - & 1.23 & \\
\hline High $(>66 \%)$ & 250 & 32.2 & 346 & 33.4 & 0.95 & 0.75 & - & 1.19 & .65 \\
\hline \multicolumn{10}{|l|}{${ }^{\wedge}$ Total Vitamin D } \\
\hline Low $(<33 \%)$ & 284 & 36.6 & 345 & 33.3 & 1.00 & & & & \\
\hline Medium (33-66\%) & 279 & 36.0 & 353 & 34.1 & 0.95 & 0.76 & - & 1.20 & \\
\hline High $(>66 \%)$ & 213 & 27.4 & 337 & 32.6 & 0.81 & 0.63 & - & 1.03 & .09 \\
\hline \multicolumn{10}{|l|}{ Cheese } \\
\hline Low $(<33 \%)$ & 193 & 24.9 & 286 & 27.6 & 1.00 & & & & \\
\hline Medium (33-66\%) & 302 & 38.9 & 337 & 32.6 & 1.31 & 1.03 & - & 1.68 & \\
\hline High $(>66 \%)$ & 281 & 36.2 & 412 & 39.8 & 0.99 & 0.77 & - & 1.26 & .71 \\
\hline \multicolumn{10}{|l|}{ Yogurt } \\
\hline Low $(<33 \%)$ & 202 & 26.0 & 347 & 33.5 & 1.00 & & & & \\
\hline Medium (33-66\%) & 278 & 35.8 & 313 & 30.2 & 1.48 & 1.16 & - & 1.88 & \\
\hline High $(>66 \%)$ & 296 & 38.1 & 375 & 36.2 & 1.31 & 1.03 & - & 1.67 & .04 \\
\hline \multicolumn{10}{|l|}{ Milk } \\
\hline Low $(<33 \%)$ & 179 & 23.1 & 264 & 25.5 & 1.00 & & & & \\
\hline Medium (33-66\%) & 292 & 37.6 & 389 & 37.6 & 1.10 & 0.86 & - & 1.42 & \\
\hline High $(>66 \%)$ & 305 & 39.3 & 382 & 36.9 & 1.14 & 0.89 & - & 1.46 & .31 \\
\hline \multicolumn{10}{|l|}{${ }^{*}$ Total Calcium } \\
\hline Low $(<33 \%)$ & 226 & 29.1 & 345 & 33.3 & 1.00 & & & & \\
\hline Medium (33-66\%) & 274 & 35.3 & 355 & 34.3 & 1.16 & 0.91 & - & 1.48 & \\
\hline High $(>66 \%)$ & 276 & 35.6 & 335 & 32.4 & 1.20 & 0.92 & 一 & 1.58 & .18 \\
\hline
\end{tabular}

All values adjusted for sex, age, study center, self-reported hypertensive status, BMI, and smoking status.

Frequency of dietary intake variables categorized into tertiles based on intake among genotypes controls.

${ }^{\wedge}$ Total Vitamin D (L, M, H) variable based on liver, egg, and salt and fresh water fish consumption.

A Also adjusted for years of occupational UV exposure.

* Total calcium variable based on cheese, yogurt, and milk consumption.

A categorical exposure matrix was used to evaluate the association between RCC risk and cumulative occupational UV exposure based on tertiles of exposure levels among male controls.
2.4. Laboratory Procedures. Genomic DNA was extracted from whole blood buffy coat using a standard phenol chloroform method at the National Cancer Institute (NCI) laboratory. Genotyping was conducted with a GoldenGate 
Oligo Pool All (OPA) assay by Illumina (www.illumina.com). DNA samples from RCC cases and controls were coded and randomized on polymerase chain reaction (PCR) plates for genotyping analyses. A random 5\% duplicate sample was selected and dispensed across plates genotyped for quality control.

As previously reported in this population [17], the ten SNPS across the VDR gene (rs11574027, rs4760648, rs2853564, rs2254210, and rs886441) and RXRA gene (rs100791, rs3118523, rs748964, rs3118536, and rs10776909) that were significantly associated with RCC risk were further evaluated in this study to see if SNPs modified associations between RCC risk and dietary or occupational UV exposure. Tag SNPs were originally selected to provide $80 \%$ to $90 \%$ coverage across the genomic regions of interest and were applicable, while nonsynonymous SNPs were selected for their putative functional significance. All SNPs selected for analysis had a variant allele frequency of at least $5 \%$ as reported by the NCI SNP500Cancer Database (http://snp500cancer.nci.nih.gov) [24] and had a validated assay at the NCI Core Genotyping Facility (CGF) (http://cgf.nci.nih.gov/home.cfm). The concordance rate between duplicate DNA samples ranged from $93 \%$ to $100 \%$ and completion rates ranged from $98 \%$ to $100 \%$. The genotype frequencies among controls did not differ from the expected Hardy-Weinberg equilibrium proportions $(P>$ $.05)$.

2.5. Statistical Analysis. The distributions of select characteristics and known RCC risk factors (sex, age, smoking habits, self-reported hypertension status, body mass index (BMI), family history of cancer, country of residence, and particular dietary variables) were compared between cases and controls using the Chi-square test. Odds ratios (ORs) and $95 \%$ confidence intervals (95\% CIs) using unconditional logistic regression were calculated to estimate the associations between individual SNPs and RCC risk.

SNP variants were assessed using a dominant model using the Wald chi-square test for the presence or absence of the variant allele $(0,1)$. Occupational UV exposure was assessed in tertiles calculated from the exposure prevalence among male controls. All regression models were adjusted for age, sex, study center, smoking status (ever, never), BMI, and self-reported hypertensive status.

$V D R$ and RXRA haplotype blocks identified previously [17] were analyzed in relation to RCC risk using Haplostats (R version 2.4.0; http://www.r-project.org) [25]. Associations between common haplotypes ( $>5 \%$ frequency) and RCC risk were evaluated by computing ORs and $95 \%$ CIs using the most common haplotype as the referent category. Interactions were tested by comparing regression models with and without interaction terms using a likelihood ratio test (LRT). Interactions between dietary components, select $V D R$ and RXRA SNPs, and RCC risk were only assessed for total calcium, total vitamin $\mathrm{D}$, and for intake frequency food groups that were found to be statistically associated with RCC risk (i.e., eggs and yogurt).
All analyses were conducted in STATA 9.0 unless otherwise specified (STATA Corporation, College Station, TX).

\section{Results}

Table 1 provides a description of genotyped study participants and known RCC risk factors. Cases and controls were comparable in age and sex; however, cases were more likely to have excess BMI $\left(>30 \mathrm{~kg} / \mathrm{m}^{2}\right)$, self-reported hypertension, and have a first degree relative with cancer. After adjustment for age, BMI, hypertension, study center, and sex the association with smoking was no longer observed [26].

Associations between intake frequency of vitamin D and calcium rich foods and RCC risk among genotyped participants are shown in Table 2 . A statistically significant reduction in RCC risk was observed with increasing consumption frequency of eggs $(P$-trend $=.02)$. A borderline statistically significant reduction in RCC risk was observed with increasing consumption frequency of total vitamin D $(P$-trend $=.09)$. In contrast, increased RCC risk was observed with increasing consumption frequency of yogurt $(P$-trend $=$ $.04)$.

Associations between RCC risk, RXRA SNPs, and intake frequency of eggs and total vitamin $\mathrm{D}$ are shown in Table 3. Inverse associations were observed between RCC risk and subjects with the wild type rs1007971 ( $P$-trend $=.05), \mathrm{rs} 3118523(P$-trend $=.01), \operatorname{rs} 748964(P$-trend $=$ $.01)$, rs3118536 ( $P$-trend $=.02$ ), and rs10776909 ( $P$-trend $=.05) R X R A$ genotypes compared to individuals with at least 1 variant allele. However, after stratification by egg intake, reduced RCC risk was observed with increasing consumption frequency of eggs among subjects with $\geq 1$ variant rs1007971 $(P$-trend $<.001)$, rs3118523 $(P$-trend $<$ $.001)$, rs748964 ( $P$-trend $<.001)$, rs3118536 ( $P$-trend < $.001)$, and rs10776909 ( $P$-trend $=.004)$ allele across the $R X R A$ gene. Interactions were only significant for $R X R A$ SNPs located downstream, $3^{\prime}$ of the coding sequence that is, rs1007971 $(P$-interaction $=.01)$, rs3118523 $(P$-interaction $=.01)$, and rs748964 $(P$-interaction $=.01)$. Although not statistically significant, a weaker inverse association between RCC risk and total vitamin D (based on liver, eggs, and fish) intake frequency was observed. No association was observed between any of the VDR SNPs and dietary consumption frequency of eggs or total vitamin D (data not shown).

Associations between RCC risk, RXRA SNPs, and intake frequency of yogurt and total calcium are shown in Table 4. A positive association was observed between RCC risk and increasing consumption frequency of yogurt among participants with the wild type $C C$ rs3118538 ( $P$-trend = $.01)$ and $C C$ rs10776909 $(P$-trend $=.01)$ alleles across the RXRA gene; rs10776909 located in intron 1 was also shown to significantly modify associations between frequency of dietary intake of yogurt and RCC risk $(P$-interaction $=.04)$. Similarly, increased RCC risk was observed with increasing total calcium intake frequency for subjects with the wild type $C C$ rs3118538 ( $P$-trend $=.05)$ and $C C$ rs10776909 $(P$ trend $=.02)$ alleles. Significant interactions were observed for both SNPs, located in introns 1 and 4, within the RXRA 


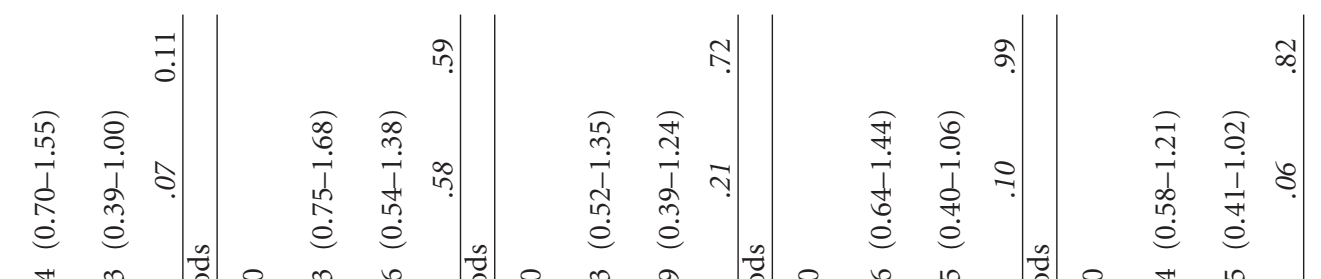

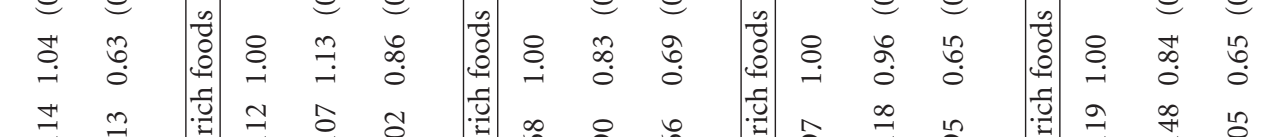

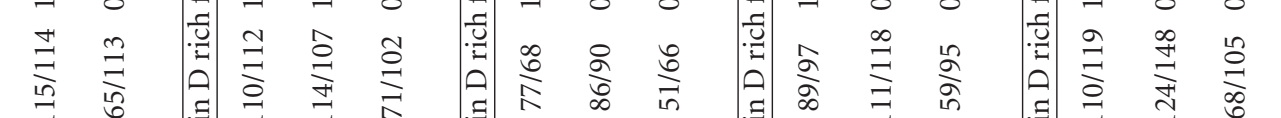

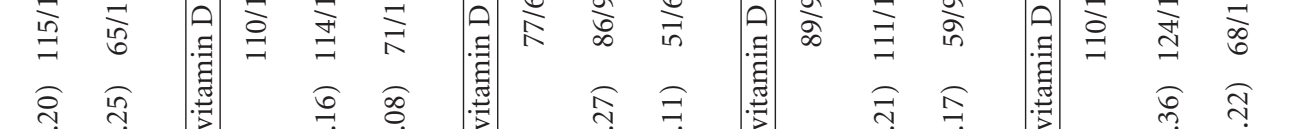

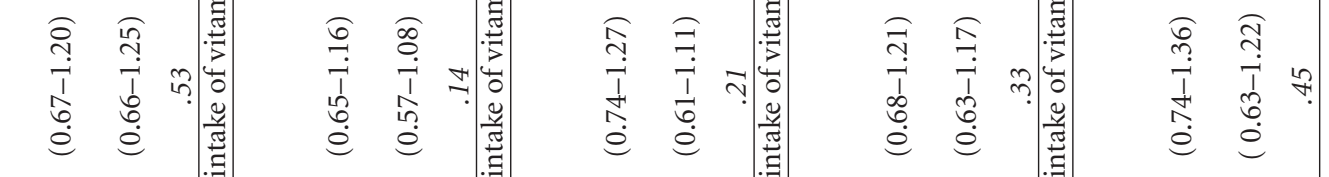

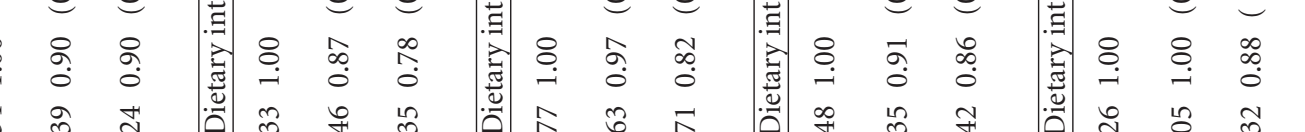

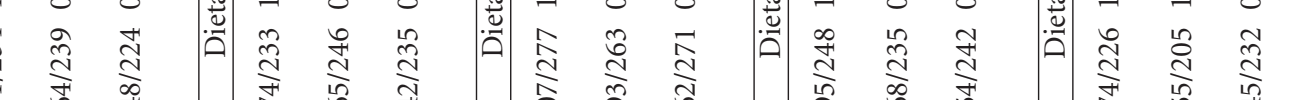

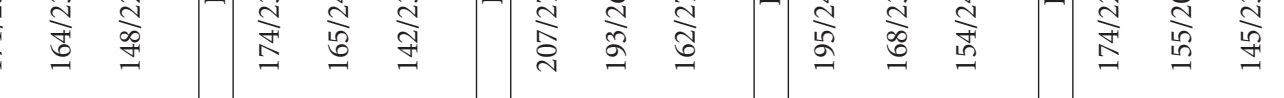

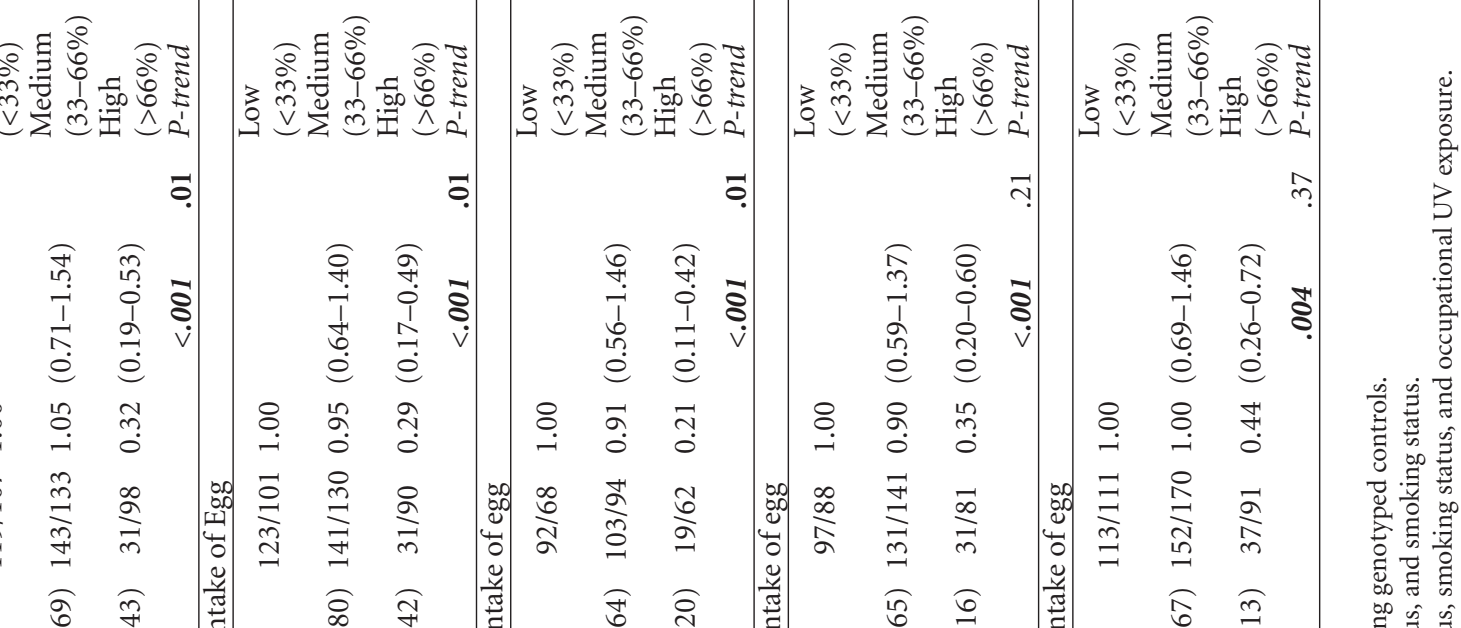

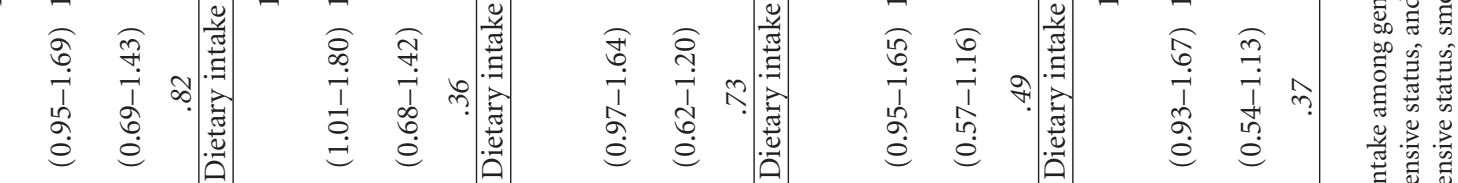

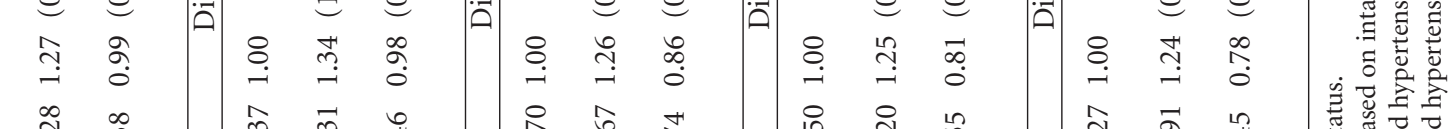

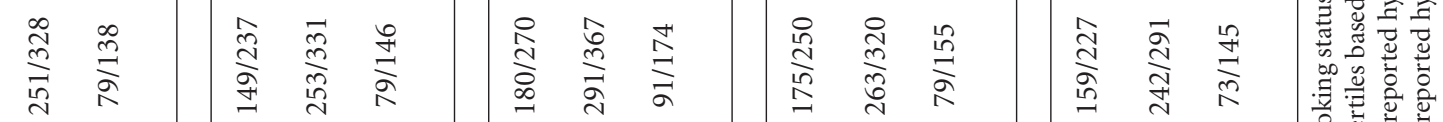

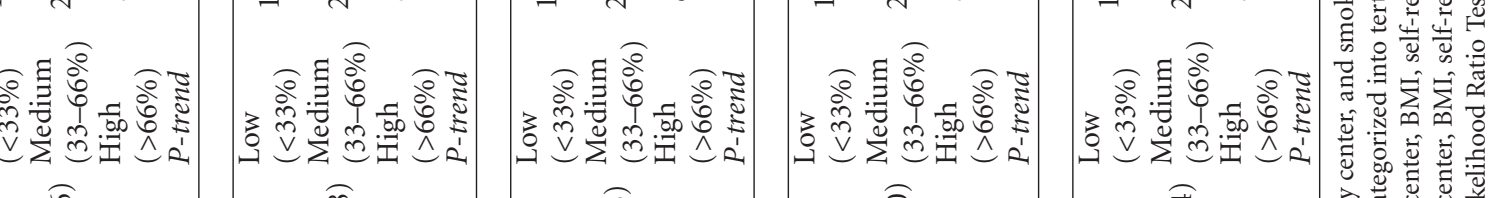

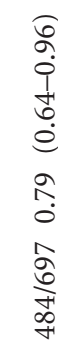

¿

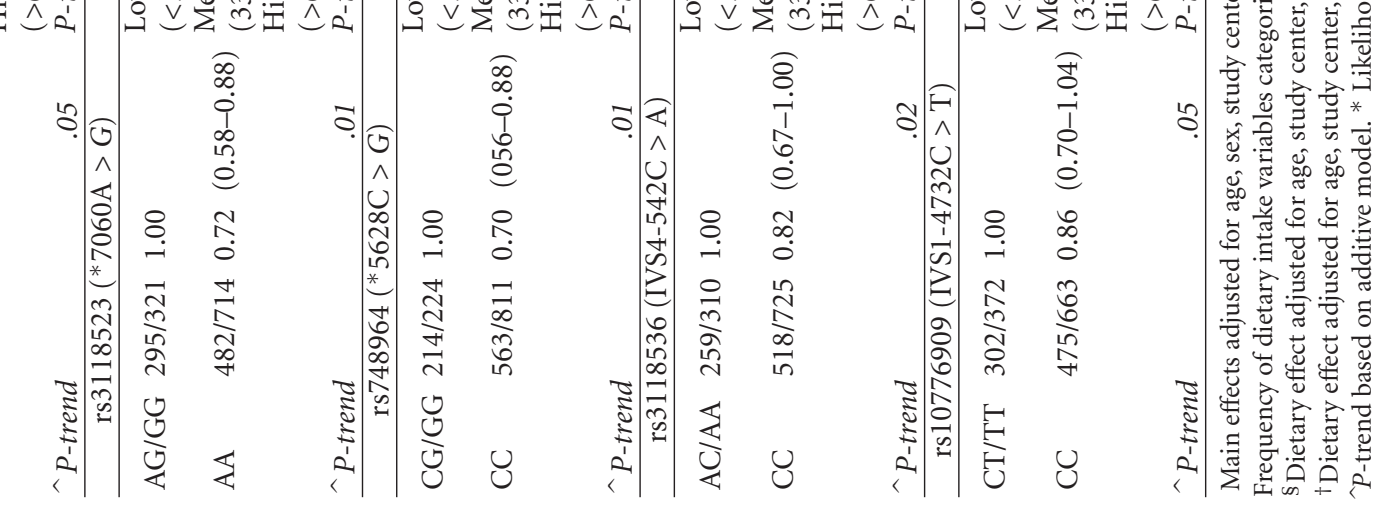


TABLE 4: Effect of RXRA SNPs on dietary intake frequency of calcium rich foods and risk of renal cell carcinoma.

\begin{tabular}{|c|c|c|c|c|c|c|c|c|c|c|c|c|c|c|}
\hline & \multicolumn{6}{|c|}{ rs3118538 (IVS4-542C > A) } & \multicolumn{8}{|c|}{ rs10776909 (IVS1-4732C > T) } \\
\hline & \multicolumn{3}{|c|}{ Wild type } & \multicolumn{3}{|c|}{$\geq 1$ variant } & \multicolumn{4}{|c|}{ Wild type } & \multicolumn{3}{|c|}{$\geq 1$ variant } & \\
\hline & \multicolumn{3}{|c|}{ Cases/Controls } & \multicolumn{3}{|c|}{ Cases/Controls } & \multicolumn{4}{|c|}{ Cases/Controls } & \multicolumn{3}{|c|}{ Cases/Controls } & \\
\hline & $\mathrm{N} / \mathrm{N}$ & OR & (UCI-LCI) & $\mathrm{N} / \mathrm{N}$ & OR & (UCI-LCI) & ${ }^{*}$ LRT & $\mathrm{N} / \mathrm{N}$ & OR & (UCI-LCI) & $\mathrm{N} / \mathrm{N}$ & OR & (UCI-LCI) & ${ }^{*}$ LRT \\
\hline \multicolumn{15}{|l|}{$\begin{array}{l}\text { Dietary Intake of } \\
\text { Yogurt }\end{array}$} \\
\hline Low $(<33 \%)$ & $133 / 266$ & 1.00 & & $77 / 91$ & 1.00 & & & $123 / 247$ & 1.00 & & $87 / 110$ & 1.00 & & \\
\hline Medium (33-66\%) & ) $185 / 212$ & 1.88 & $(1.34-2.64)$ & $85 / 91$ & 1.23 & $(0.73-2.08)$ & & $169 / 198$ & 1.84 & $(1.29-2.63)$ & $101 / 105$ & 51.29 & $(0.81-2.07)$ & \\
\hline $\operatorname{High}(>66 \%)$ & $199 / 247$ & 1.69 & $(1.18-2.42)$ & $97 / 128$ & 0.87 & $(0.51-1.50)$ & & $182 / 218$ & 1.71 & $(1.18-2.49)$ & $114 / 157$ & 70.94 & $(0.58-1.54)$ & \\
\hline \multirow{2}{*}{$P$-trend } & & & .01 & & & .41 & & & & .01 & & & .58 & \\
\hline & & & & & & & .09 & & & & & & & .04 \\
\hline \multicolumn{15}{|l|}{$\begin{array}{l}\text { Dietary Intake of } \\
\text { Calcium Rich } \\
\text { Foods }\end{array}$} \\
\hline Low $(<33 \%)$ & $146 / 248$ & 1.00 & & $80 / 97$ & 1.00 & & & $135 / 236$ & 1.00 & & $91 / 109$ & 1.00 & & \\
\hline Medium (33-66\%) & ) $180 / 261$ & 1.18 & $(0.88-1.59)$ & $94 / 94$ & 1.22 & $(0.78-1.89)$ & & $162 / 238$ & 1.21 & $(0.89-1.66)$ & $112 / 117$ & 71.10 & $(0.73-1.64)$ & \\
\hline $\operatorname{High}(>66 \%)$ & $191 / 216$ & 1.39 & $(1.00-1.93)$ & $85 / 119$ & 0.76 & $(0.47-1.22)$ & & $177 / 189$ & 1.49 & $(1.06-2.11)$ & $99 / 146$ & 0.73 & $(0.47-1.14)$ & \\
\hline \multirow[t]{2}{*}{ P-trend } & & & .05 & & & .25 & & & & .02 & & & .16 & \\
\hline & & & & & & & .03 & & & & & & & .02 \\
\hline
\end{tabular}

Dietary effect adjusted for age, sex, study center, BMI, self-reported hypertensive status, and smoking status.

Frequency of dietary intake variables categorized into tertiles based on intake among genotyped controls.

Calcium rich foods based on consumption of cheese, yogurt, and milk.

Main effects adjusted for age, sex, study center, and smoking status.

rs3118538 (IVS4-542C > A) main effects: AC (0.68(0.39-1.18)), AA (0.58(0.34-0.99)), $P$-trend $=.02$; AC/AA $(0.82(0.67-1.00))$.

rs10776909 (IVS1-4732C > T) main effects: CT $(0.69(0.42-1.13))$, TT $(0.62(0.38-1.00)), P$-trend $=.05$; CT/TT $(0.86(0.70-1.04))$.

*Likelihood Ratio Test.

gene. No association was observed between intake frequency of yogurt or total calcium and the other SNPs $(\mathrm{N}=8)$ previously shown to be associated with RCC risk in this study [17].

Previously we observed a significant reduction (38\%) in RCC risk among male participants occupational exposed to UV [18]. In this study, neither VDR nor RXRA SNPs modified the observed associations (data not shown); however, certain VDR haplotypes, previously associated with RCC risk in this study [18], were found to modify associations between RCC risk and occupational UV exposure among male participants (Table 5). Males with the VDR A-G-C (rs2254210, rs2853564, and rs4760648) haplotype were at a significantly increased $(\mathrm{OR}=1.27 ; 95 \% \mathrm{CI}=1.03-1.57)$ risk of renal cancer compared to males with the most common referent haplotype $G-A-T$. When haplotypes were stratified by occupational UV category, only males in the highest UV exposure category with the same $V D R$ haplotype, $A-G-C$, were shown to have a significantly stronger increase in RCC risk $(\mathrm{OR}=1.70 ; 95 \% \mathrm{CI}=1.14-2.53)$ compared to males with the referent haplotype, $G-A-T$. No associations between $V D R$ haplotypes, RCC risk and cumulative occupational UV exposure were observed among male participants in the lowest or middle UV category. A borderline significant interaction was also observed between VDR haplotypes, occupational UV exposure, and RCC risk ( $P$-interaction $=$ $.06)$.

\section{Discussion}

In this study, reduced RCC risk was observed with increasing consumption frequency of eggs while increased RCC risk was observed with increasing consumption frequency of yogurt. After stratification by VDR and RXRA genotypes, the two genes previously associated with RCC risk in this study [17], only RXRA gene variants modified associations between RCC risk and consumption frequency of eggs, yogurt, and total calcium. RXRA SNPs $3^{\prime}$ of the coding sequence modified the association for intake frequency of eggs while intronic RXRA SNPS modified the association for intake frequency of yogurt and total calcium. No associations with VDR or RXRA genotypes were observed with occupational UV exposure and RCC risk among male participants previously observed to have lower RCC risk with increasing occupational sunlight exposure [18]; however, when $V D R$ haplotypes were examined male subjects in the highest cumulative occupational UV exposure category with the $A-G-C V D R$ haplotype had a significant increase in RCC risk compared to male subjects with the referent $G-A-T$ haplotype.

Numerous studies have established a link between diet and cancer risk or progression, therefore the World Cancer Research Fund has recently acknowledged that after smoking, diet may be the second most important contributor to the global burden of cancer [27]. While, dietary vitamin $\mathrm{D}$ intake constitutes a small proportion of total vitamin D 


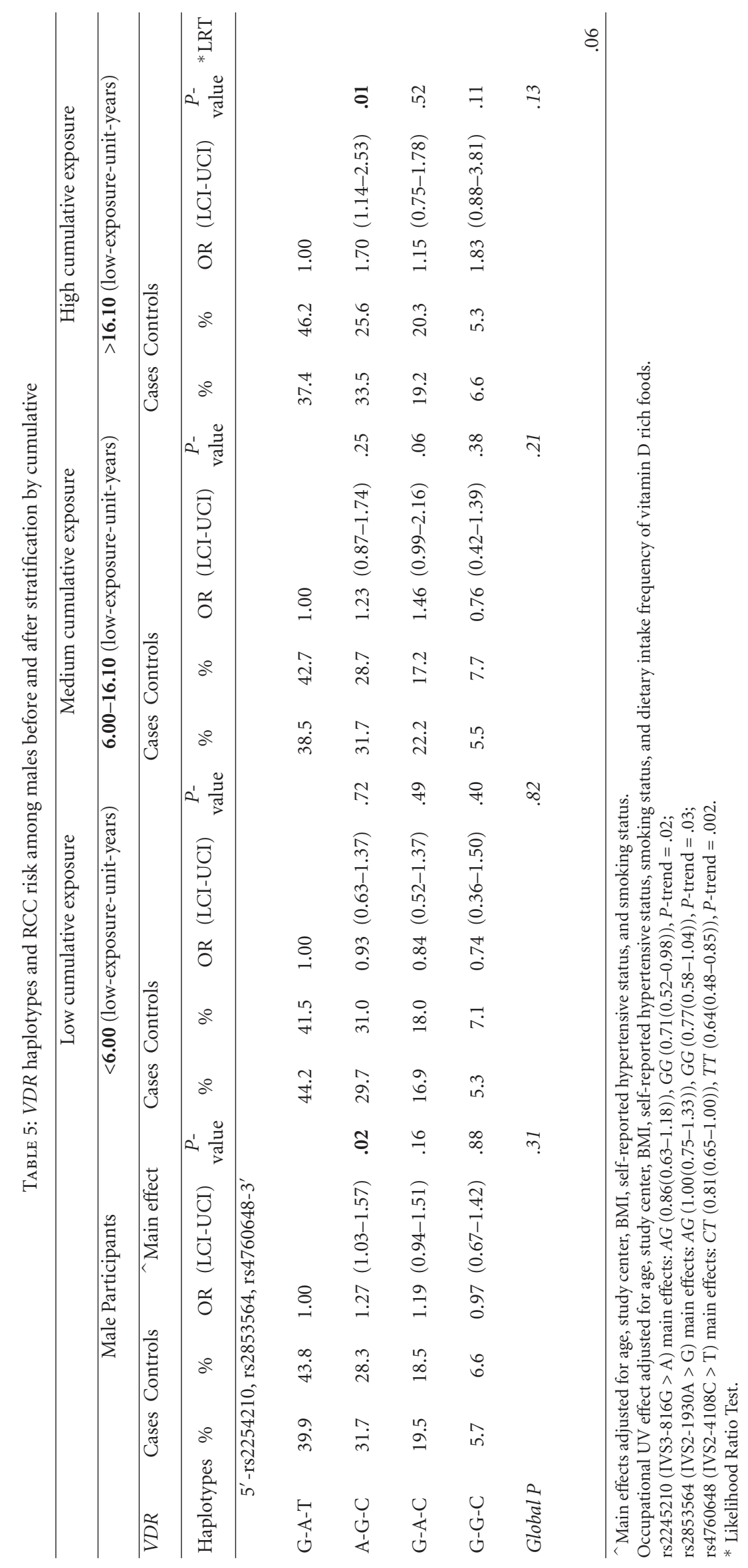


requirements $[8,9]$, studies suggest that dietary intake of vitamin D may play an important role in determining cancer risk $[5,28]$. This is particularly important in areas where ultraviolet levels of exposure vary seasonally. Overall, most epidemiological studies investigating the association between RCC risk and vitamin D or calcium rich foods have been inconsistent and generally null $[23,28-32]$. Similar to the results we present in this study, an Italian case-control study of 767 RCC cases and 1534 controls observed a significant inverse association between dietary vitamin $\mathrm{D}$ intake, based on a 78-item food frequency questionnaire, and RCC risk [5]. However, an earlier Canadian RCC case-control study showed no association for intake of individual foods rich in vitamin D (i.e., fish and eggs) or calcium (i.e., milk, diary, and cheese) [28]. In contrast, calcium supplement intake in that study was shown to significantly reduce RCC risk with increasing the number of years of intake [28].

While no reports to date have investigated the association between RCC risk, dietary intake of vitamin D or calcium rich foods, and vitamin $\mathrm{D}$ gene variants, results have been reported for VDR SNPs and other cancers. For example, two colorectal case-control studies, one in the US [33] and one in UK [34], observed reduced cancer risk with increasing intake of vitamin $\mathrm{D}$ and calcium rich foods among participants with the homozygous VDR variant (polyA, BsmI, and/or ApaI) alleles compared to participants with the wild type alleles. In contrast, a US case-control study reported increased colorectal cancer risk with increasing vitamin D intake for subjects with the homozygous variant VDR (BsmI $B B$ ) allele [35]. No association between vitamin $\mathrm{D}$ intake, VDR polymorphisms (TaqI, BsmI, FokI, ApaI, and/or polyA) and lymphoma [36] and breast cancer risk [37], was reported in two separate US studies.

Epidemiological studies investigating whether polymorphisms in vitamin D pathway genes modify the relationship between cancer risk and UV exposure have been inconsistent. Similar to our results, an association between UV exposure and $V D R$ haplotypes comprised of the rs2254210 SNP was reported in a recent British prostate cancer case-control study. Four VDR SNPs in two haplotype block regions were shown to significantly increase cancer risk among men with low UV exposure [38]. However, inverse associations were reported in this population when five other VDR SNPs were examined in relation to UV exposure and prostate cancer risk [39]. Likewise, in a US case-control study of prostate cancer, reduced cancer risk was observed among subjects with the FokI and TaqI FFtt haplotype among subjects in the highest UV exposure category [40].

Our study adds to the limited epidemiological evidence that calcium and vitamin $\mathrm{D}$ (from dietary intake or UV exposure), and VDR and RXRA vitamin D pathway genes may play a role in RCC etiology. The biological activity of vitamin $\mathrm{D}$ is mediated by a high-affinity receptor, VDR, which acts as a ligand-activated transcription factor that forms a heterodimer with $R X R[13,15,16,41]$. This $V D R$ $R X R$ heterodimer complex is directed to the vitamin $\mathrm{D}$ responsive element in the promoter region of 1,25-regulated genes $[13,15,16,41]$. Different polymorphisms in the $V D R$ gene have been speculated to result in variation of
$V D R$ expression and result in changes to circulating levels of active vitamin D [4]. For this reason, epidemiological studies suggest that tissue specific expression of vitamin D pathway genes function as the primary mechanism involved in linking vitamin $\mathrm{D}$ status with anticarcinogenic effects of $1,25(\mathrm{OH})_{2} \mathrm{D}_{3}$ [42]; therefore, lower renal cancer risk may be associated with higher circulating levels of $25(\mathrm{OH}) \mathrm{D}$, the storage form of vitamin $\mathrm{D}$ by providing substrate for renal tissue-specific synthesis of $1,25(\mathrm{OH})_{2} \mathrm{D}_{3}$ [43]. RXRA on the other hand may play a critical role in vitamin D activity, particularly from dietary sources, since this gene has been shown to regulate cholesterol [44], which is abundant in eggs and yogurt, the food groups found statistically associated with renal cancer risk in this study. RXRA regulated fatty acid and cholesterol metabolism through intestinal cholesterol absorption and bile acid synthesis [44]. Cholesterol metabolism has been associated with arthrosclerosis which is associated with hypertension and cardiovascular risk, known risk factors of RCC [45].

Strengths of our study include the use of HapMap to tag genes of interest using high (80-90\%) genomic coverage both $5^{\prime}$ and $3^{\prime}$ of the target genes. This study also observed high participation rates among newly diagnosed histologically confirmed cases and collected biologic materials from a high proportion of subjects. The large sample size of this study provided sufficient statistical power to detect relatively small associations; however, the power to detect gene-environment interactions was limited and results need to be interpreted cautiously.

Inherent limitations of our study include (1) the possibility of nondifferential dietary recall bias, (2) possible misclassification of vitamin D and calcium intake, (3) the inability to control for potential confounders such as total caloric intake, which may bias results away from the null, (4) lack of data regarding dietary vitamin D or calcium supplements, and (5) limited statistical power for geneenvironment interactions. Additionally, $18.7 \%$ of controls were selected among patients admitted with gastrointestinal conditions, which may potentially influenced dietary habits thus, affecting study results. Limitations stemming from the estimate of occupational UV exposure [18], such as nondifferential, inaccurate, or incomplete recall of all occupational histories may also have biased results. While hospital-based case-control studies have potential limitations due to possible differences with population controls, these studies can improve response rates for the intense collection of biological specimens and therefore reduce the chances of bias in the assessment of gene-environment interactions [46]. Although there is general agreement that the serum $25(\mathrm{OH})$ vitamin $\mathrm{D}$ level is the best indicator of current vitamin $\mathrm{D}$ status, the biochemical marker has a short halflife and a single measurement of $25(\mathrm{OH})$ vitamin D may not reflect long-term status that would be important in studies of cancer. Moreover, vitamin D status postdiagnosis among cases may reflect disease rather than historical vitamin D levels of participants when they were healthy. Lastly, while global $P$-values were assessed previously on these genes [17], we cannot completely rule out the possibility of false positive findings in this study due to multiple comparisons. 
In conclusion, common variants in $V D R$ and $R X R A$ genes were shown to modify associations between RCC risk and consumption frequency of vitamin D and calcium rich foods, and occupational UV exposure. Additional studies with large sample sizes and thorough genomic coverage of $V D R$ and RXRA pathway genes, sufficiently powered to investigate gene-environment interactions, are needed to confirm these results and to provide further insight into the role of calcium and vitamin D in RCC etiology.

\section{References}

[1] W. H. Chow and S. S. Devesa, "Contemporary epidemiology of renal cell cancer," Cancer Journal, vol. 14, no. 5, pp. 288-301, 2008.

[2] L. Lipworth, R. E. Tarone, and J. K. McLaughlin, "The epidemiology of renal cell carcinoma," Journal of Urology, vol. 176, no. 6, part 1, pp. 2353-2358, 2006.

[3] B. I. Rini, S. C. Campbell, and W. K. Rathmell, "Renal cell carcinoma," Current Opinion in Oncology, vol. 18, no. 3, pp. 289-296, 2006.

[4] T. Ikuyama, T. Hamasaki, H. Inatomi, T. Katoh, T. Muratani, and T. Matsumoto, "Association of vitamin D receptor gene polymorphism with renal cell carcinoma in Japanese," Endocrine Journal, vol. 49, no. 4, pp. 433-438, 2002.

[5] C. Bosetti, L. Scotti, L. D. Maso, et al., "Micronutrients and the risk of renal cell cancer: a case-control study from Italy," International Journal of Cancer, vol. 120, no. 4, pp. 892-896, 2007.

[6] W. Obara, Y. Suzuki, K. Kato, S. Tanji, R. Konda, and T. Fujioka, "Vitamin D receptor gene polymorphisms are associated with increased risk and progression of renal cell carcinoma in a Japanese population," International Journal of Urology, vol. 14, no. 6, pp. 483-487, 2007.

[7] S. Karami, P. Brennan, R. J. Hung, et al., "Vitamin D receptor polymorphisms and renal cancer risk in Central and Eastern Europe," Journal of Toxicology and Environmental Health A, vol. 71, no. 6, pp. 367-372, 2008.

[8] M. F. Holick, "Vitamin D: its role in cancer prevention and treatment," Progress in Biophysics and Molecular Biology, vol. 92, no. 1, pp. 49-59, 2006.

[9] M. F. Holick, "The vitamin D epidemic and its health consequences," Journal of Nutrition, vol. 135, no. 11, pp. 2739S-2748S, 2005.

[10] C. Garland, R. B. Shekelle, E. Barrett-Connor, M. H. Criqui, A. H. Rossof, and O. Paul, "Dietary vitamin D and calcium and risk of colorectal cancer: a 19-year prospective study in men," The Lancet, vol. 1, no. 8424, pp. 307-309, 1985.

[11] D. L. Trump, P. A. Hershberger, R. J. Bernardi, et al., "Antitumor activity of calcitriol: pre-clinical and clinical studies," The Journal of Steroid Biochemistry and Molecular Biology, vol. 89-90, no. 1-5, pp. 519-526, 2004.

[12] P. Ordonez-Moran, M. J. Larriba, N. Pendas-Franco, O. Aguilera, J. M. Gonzalez-Sancho, and A. Munoz, "Vitamin D and cancer: an update of in vitro and in vivo data," Frontiers in Bioscience, vol. 10, pp. 2723-2749, 2005.

[13] J. M. Valdivielso and E. Fernandez, "Vitamin D receptor polymorphisms and diseases," Clinica Chimica Acta, vol. 371, no. 1-2, pp. 1-12, 2006.

[14] O. Hino, T. Kobayashi, S. Momose, Y. Kikuchi, H. Adachi, and K. Okimoto, "Renal carcinogenesis: genotype, phenotype and dramatype,” Cancer Science, vol. 94, no. 2, pp. 142-147, 2003.
[15] M. R. Walters, "Newly identified actions of the vitamin D endocrine system," Endocrine Review, vol. 13, no. 4, pp. 719764, 1992.

[16] F. Thibault, G. Cancel-Tassin, and O. Cussenot, "Low penetrance genetic susceptibility to kidney cancer," BJU International, vol. 98, no. 4, pp. 735-738, 2006.

[17] S. Karami, P. Brennan, P. Stewart, et al., "Analysis of SNPs and haplotypes in VDR pathway genes and renal cancer risk," PLoS ONE. In press.

[18] S. Karami, P. Boffetta, P. Stewart, et al., "Occupational sunlight exposure and risk of renal cell carcinoma," Cancer. In press.

[19] International Agency for Research on Cancer, Globacan, April 2008, http://www-dep.iarc.fr.

[20] R. J. Hung, L. Moore, P. Boffetta, et al., "Family history and the risk of kidney cancer: a multicenter case-control study in Central Europe," Cancer Epidemiology Biomarkers and Prevention, vol. 16, no. 6, pp. 1287-1290, 2007.

[21] G. Scélo, V. Constantinescu, I. Csiki, et al., "Occupational exposure to vinyl chloride, acrylonitrile and styrene and lung cancer risk," Cancer Causes and Control, vol. 15, no. 5, pp. 445452, 2004.

[22] M. Hashibe, P. Boffetta, D. Zaridze, et al., "Contribution of tobacco and alcohol to the high rates of squamous cell carcinoma of the supraglottis and glottis in Central Europe," American Journal of Epidemiology, vol. 165, no. 7, pp. 814-820, 2007.

[23] C. C. Hsu, W. H. Chow, P. Boffetta, et al., "Dietary risk factors for kidney cancer in Eastern and Central Europe," American Journal of Epidemiology, vol. 166, no. 1, pp. 62-70, 2007.

[24] B. R. Packer, M. Yeager, L. Burdett, et al., "SNP500Cancer: a public resource for sequence validation, assay development, and frequency analysis for genetic variation in candidate genes," Nucleic Acids Research, vol. 34, database issue, pp. D617-D621, 2006.

[25] R Project for Statistical Computing, March 2008, http://www.r-project.org.

[26] J. D. Hunt, O. L. van der Hel, G. P. McMillan, P. Boffetta, and P. Brennan, "Renal cell carcinoma in relation to cigarette smoking: meta-analysis of 24 studies," International Journal of Cancer, vol. 114, no. 1, pp. 101-108, 2005.

[27] The Word Cancer Resarch Fund, June 2009, http://www.wcrf-uk.org/preventing cancer/index.php.

[28] J. Hu, Y. Mao, and K. White, "Diet and vitamin or mineral supplements and risk of renal cell carcinoma in Canada," Cancer Causes Control, vol. 14, no. 8, pp. 705-714, 2003.

[29] B. Rashidkhani, A. Akesson, P. Lindblad, and A. Wolk, "Major dietary patterns and risk of renal cell carcinoma in a prospective cohort of Swedish women," Journal of Nutrition, vol. 135, no. 7, pp. 1757-1762, 2005.

[30] J. Hu, C. La Vecchia, M. DesMeules, E. Negri, and L. Mery, "Meat and fish consumption and cancer in Canada," Nutrition and Cancer, vol. 60, no. 3, pp. 313-324, 2008.

[31] F. Bravi, C. Bosetti, L. Scotti, et al., "Food groups and renal cell carcinoma: a case-control study from Italy," International Journal of Cancer, vol. 120, no. 3, pp. 681-685, 2007.

[32] A. Wolk, S. C. Larsson, J. E. Johansson, and P. Ekman, "Longterm fatty fish consumption and renal cell carcinoma incidence in women," Journal of the American Medical Association, vol. 296, no. 11, pp. 1371-1376, 2006.

[33] M. L. Slattery, S. L. Neuhausen, M. Hoffman, et al., "Dietary calcium, vitamin D, VDR genotypes and colorectal cancer," International Journal of Cancer, vol. 111, no. 5, pp. 750-756, 2004. 
[34] R. A. Hubner, K. R. Muir, J. F. Liu, et al., "Dairy products, polymorphisms in the vitamin $\mathrm{D}$ receptor gene and colorectal adenoma recurrence," International Journal of Cancer, vol. 123, no. 3, pp. 586-593, 2008.

[35] H. S. Kim, P. A. Newcomb, C. M. Ulrich, et al., "Vitamin D receptor polymorphism and the risk of colorectal adenomas: evidence of interaction with dietary vitamin D and calcium," Cancer Epidemiology Biomarkers and Prevention, vol. 10, no. 8, pp. 869-874, 2001.

[36] M. P. Purdue, P. Hartge, S. Davis, et al., "Sun exposure, vitamin $\mathrm{D}$ receptor gene polymorphisms and risk of non-Hodgkin lymphoma," Cancer Causes and Control, vol. 18, no. 9, pp. 989999, 2007.

[37] M. L. McCullough, V. L. Stevens, W. R. Diver, et al., "Vitamin D pathway gene polymorphisms, diet, and risk of postmenopausal breast cancer: a nested case-control study," Breast Cancer Research, vol. 9, no. 1, article R9, 2007.

[38] N. J. Rukin, C. Luscombe, S. Moon, et al., "Prostate cancer susceptibility is mediated by interactions between exposure to ultraviolet radiation and polymorphisms in the $5^{\prime}$ haplotype block of the vitamin D receptor gene," Cancer Letters, vol. 247, no. 2, pp. 328-335, 2007.

[39] S. Moon, S. Holley, D. Bodiwala, et al., "Associations between $\mathrm{G} / \mathrm{A}^{1229}, \mathrm{~A} / \mathrm{G}^{3944}, \mathrm{~T} / \mathrm{C}^{30875}, \mathrm{C} / \mathrm{T}^{48200}$ and $\mathrm{C} / \mathrm{T}^{65013}$ genotypes and haplotypes in the vitamin $\mathrm{D}$ receptor gene, ultraviolet radiation and susceptibility to prostate cancer," Annals of Human Genetics, vol. 70, no. 2, pp. 226-236, 2006.

[40] E. M. John, G. G. Schwartz, J. Koo, D. Van den Berg, and S. A. Ingles, "Sun exposure, vitamin D receptor gene polymorphisms, and risk of advanced prostate cancer," Cancer Research, vol. 65, no. 12, pp. 5470-5479, 2005.

[41] A. J. Brown, A. Dusso, and E. Slatopolsky, "Vitamin D," American Journal of Physiology, vol. 227, no. 2, part 2, pp. F157-F175, 1999.

[42] K. Townsend, C. M. Banwell, M. Guy, et al., "Autocrine metabolism of vitamin $\mathrm{D}$ in normal and malignant breast tissue," Clinical Cancer Research, vol. 11, no. 9, pp. 3579-3586, 2005.

[43] M. L. McCullough, V. L. Stevens, W. R. Diver, et al., "Vitamin D pathway gene polymorphisms, diet, and risk of postmenopausal breast cancer: a nested case-control study," vol. 9, no. 1, article R9, 2007.

[44] R. A. Hegele and H. Cao, "Single nucleotide polymorphisms of RXRA encoding retinoid X receptor alpha," Journal of Human Genetics, vol. 46, no. 7, pp. 423-425, 2001.

[45] G. L. Bakris and E. Ritz, "The message for World Kidney Day 2009: hypertension and kidney disease-a marriage that should be prevented," Journal of Hypertension, vol. 27, no. 3, pp. 666-669, 2009.

[46] S. Wacholder, N. Chatterjee, and P. Hartge, "Joint effect of genes and environment distorted by selection biases: implications for hospital-based case-control studies," Cancer Epidemiology Biomarkers and Prevention, vol. 11, no. 9, pp. 885-889, 2002. 


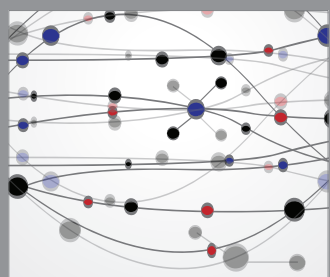

The Scientific World Journal
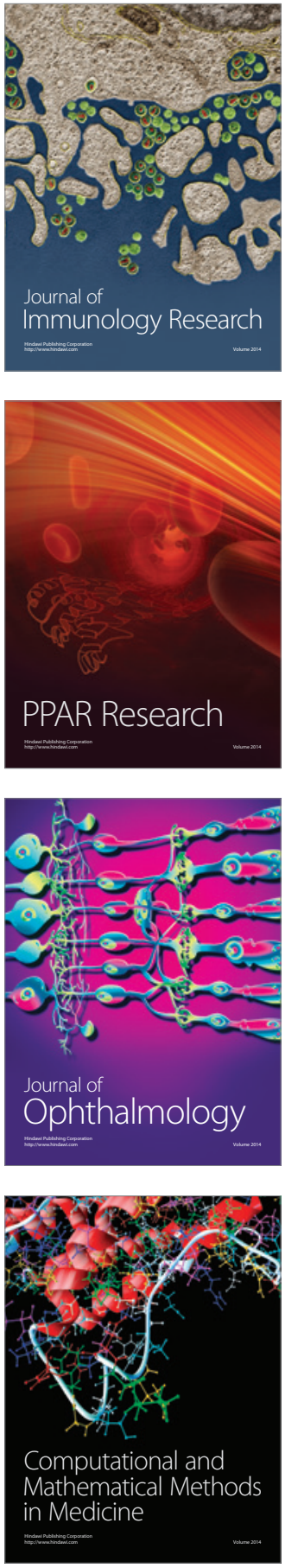

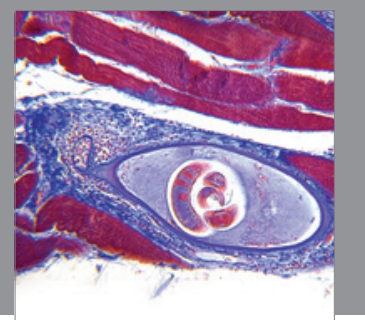

Gastroenterology

Research and Practice
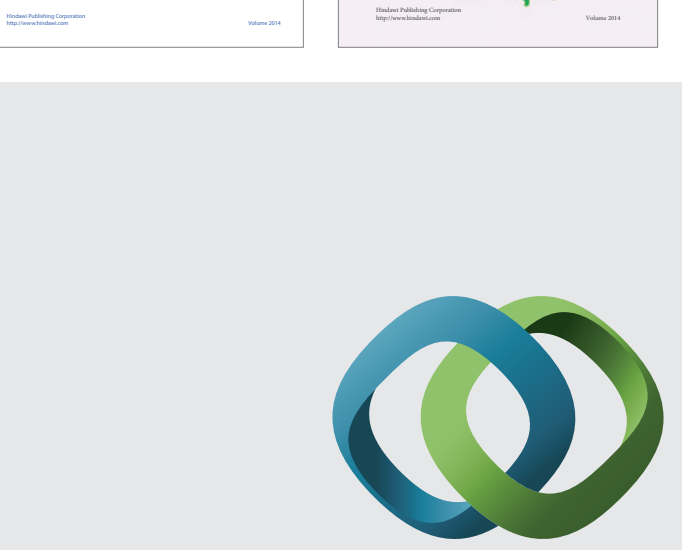

\section{Hindawi}

Submit your manuscripts at

http://www.hindawi.com
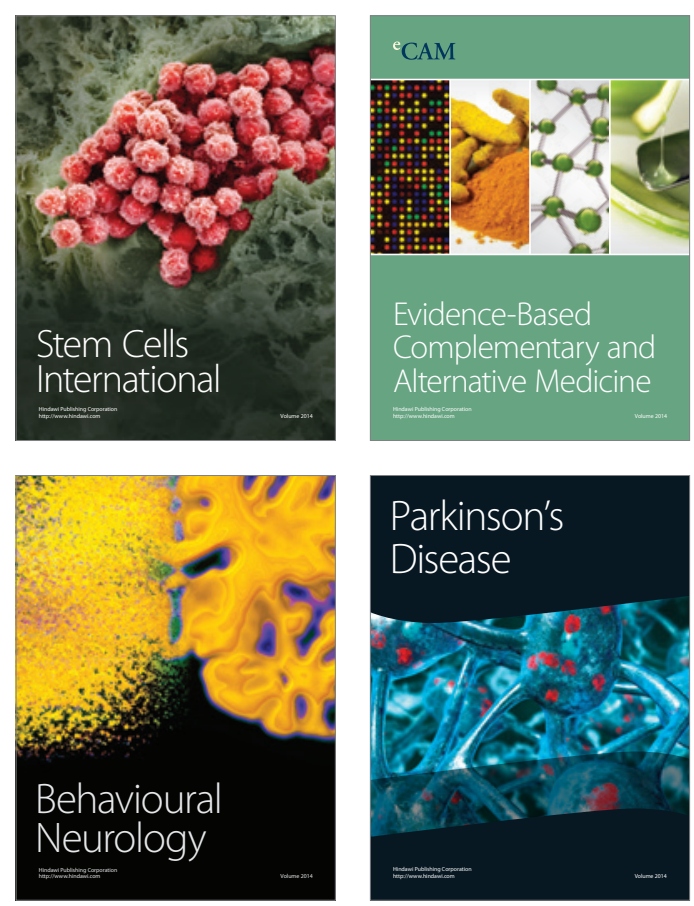

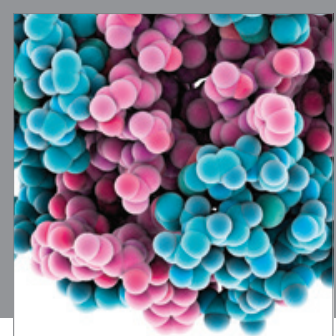

Journal of
Diabetes Research

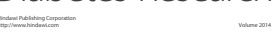

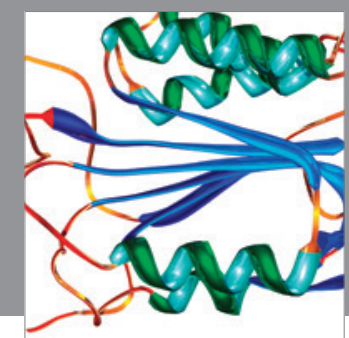

Disease Markers
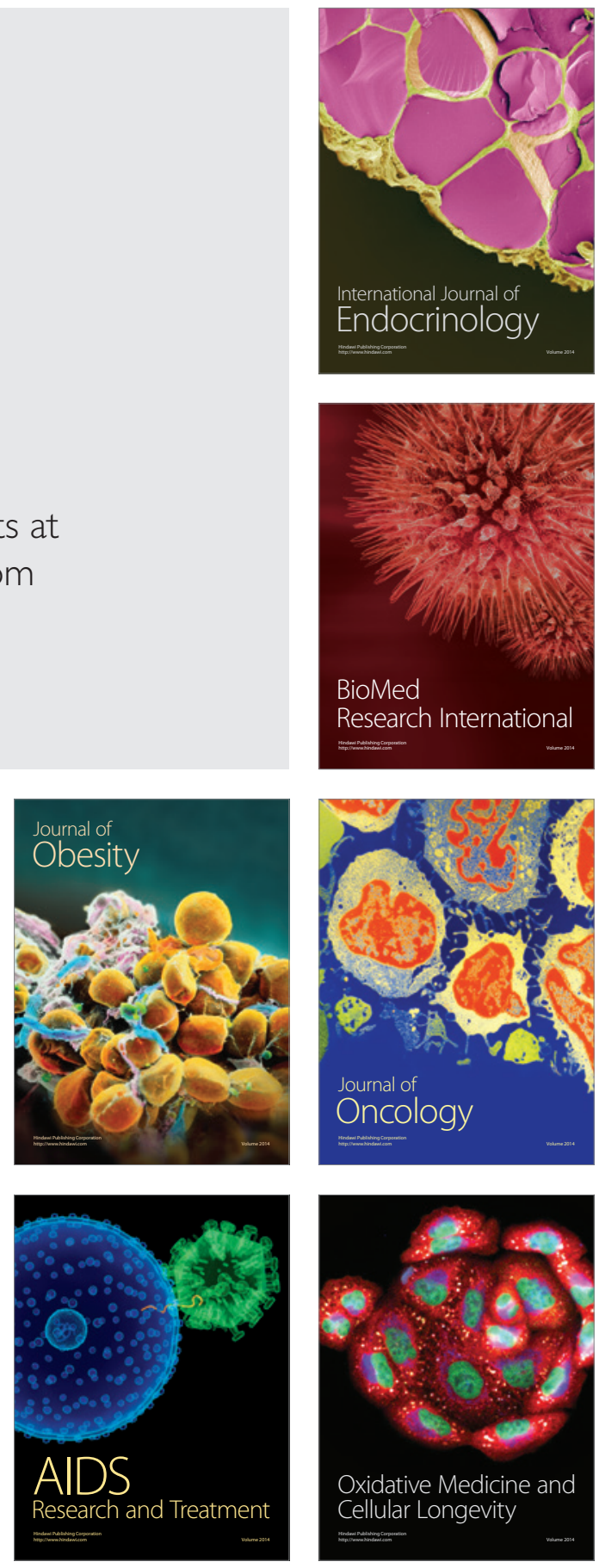